\section{LUNG}

\section{Breathing again after injury}

Parabronchial smooth muscle cell (PSMC) progenitors prompt epithelial proliferation by secreting fibroblast growth factor 10 (Fgf10) during lung development. A new study shows that these cells also act as a self-renewal niche after airway epithelium injury, leading to lung repair in adult mice (J. Clin. Invest. doi:10.1172/JCI58097).

Thomas Volckaert and his colleagues found that treatment of mice with naphthalene leads to the injury (or loss) of Clara epithelial cells. In response, Wnt signaling is reactivated in mature PSMCs, which secrete Fgf10 that then activates surviving variant Clara cells in a paracrine manner, allowing these cells to help replace epithelium. Fgf10-mediated activation induces Snail1 expression and Notch activation in the Clara cells, resulting in an epithelial-to-mesenchymal transition that promotes lung epithelium repair and may indicate an epithelial stem cell-like state.

Fgf10 from the PMSC niche may therefore be involved in lung development and airway epithelium regeneration after injury. Further investigation will be required to elucidate the role of Wnt and Fgf10 signaling during abnormal repair and remodeling in people suffering from chronic injury to these tissues, such as in asthma. $-C P$

\section{NEUROSCIENCE}

\section{A common toxic expansion}

A six-nucleotide-repeat expansion in the gene C9ORF72 links the two distinct neurodegenerative diseases frontotemporal dementia (FTD) and amyotrophic lateral sclerosis (ALS), two groups report in Neuron.

FTD is characterized by cognitive dysfunction caused by neuron death at the surface of the brain, whereas ALS is a motor disease caused by neuron death in areas of the brain and spinal cord that control motion. These two diseases can occur together in individuals and in families, suggesting they have a common cause.

Linkage studies looking at multiple families with ALS, FTD or both previously associated a small region on chromosome 9 with these diseases in some families, but the causal gene was unknown. Now, using deep sequencing in affected families, Alan Renton et al. (doi:10.1016/j.neuron.2011.09.010) and Mariely DeJesus-Hernandez et al. (doi:10.1016/j.neuron.2011.09.011) found a

\title{
METABOLISM \\ Orexin acts on brown fat
}

Deficiency in orexin neuropeptide hormones has been linked to narcolepsy, a sleep disorder. People with narcolepsy are more likely to be obese, but how this occurs is unclear, as they do not eat more or exercise less than normal individuals. A recent study (Cell Metab. 14, 478-489) suggests that orexin's effects on metabolism might be mediated by regulating brown adipose tissue (BAT).

Dyan Sellayah et al. found that wild-type mice undergo a compensatory increase in metabolic rate in response to a high-fat diet, but orexin-null narcoleptic mice failed to adapt their metabolism accordingly. The authors showed that this is because orexin is required for the differentiation of brown adipocyte precursors. An absence of orexin impaired the thermogenic capacity of brown fat, which can normally function as a mechanism to counteract weight gain. The BAT developmental defect in orexin-null neonates could be rescued by injecting orexin to their orexin-null mothers.

Although orexin is unlikely to be a therapeutic option for treating obesity, due to its complex effects on the brain, further investigation of the pathways controlled by orexin in BAT may allow the discovery of new therapeutic targets based on BAT expansion, activation or both. $-M S$

GGGGCC repeat in the first intron of C9ORF72. Unaffected individuals harbored less than 20 repeats in their C9ORF72 gene, whereas subjects with either or both diseases had up to 1,600 repeats. This repeat expansion was also found in affected individuals without familial disease. From their analysis, the researchers argued that the repeat expansion seemed to be the most common genetic cause of ALS and FTD identified so far.

Although the function of C9ORF72 is unknown, its transcript can be alternatively spliced into three different variants. DeJesusHernandez et al. found that the expression of variant 1 is low in people with the repeat expansion and that C9ORF72 mRNA aggregates in the cell. However, further work will be needed to determine how these or other mechanisms cause neuron death in FTD and ALS. - EC

\section{FIBROSIS}

\section{Fighting fibrosis}

Lung fibrosis is a progressive, deadly condition for which there is no therapy. A recent clinical trial (N. Engl. J. Med. 365, 1079-1087) with a broad-spectrum tyrosine kinase receptor inhibitor reports encouraging results for people with this disease.

Luca Richeldi et al. tested the efficacy and safety of BIBF 1120, a molecule that inhibits several members of the vascular endothelial, platelet-derived and fibroblast growth factor receptor families, in a phase 2, 12-month trial in 432 patients with idiopathic pulmonary fibrosis. They found that, in people receiving the highest dose of the drug (150 mg twice a day), the rate of decline in lung function decreased by nearly $70 \%$. Similarly, there were fewer acute exacerbations of the disease in these patients compared with the placebo group.

Unfortunately, the incidence of gastrointestinal and hepatic side effects that led to discontinuation of the treatment was also more frequent for the group receiving the highest dose of the compound. The evidence of this molecule's efficacy is nevertheless heartening, as it shows that tyrosine kinase receptor inhibition may indeed be an effective therapeutic strategy against lung fibrosis. This study should therefore fuel the search for safer broadspectrum antagonists as potential fibrosis therapeutics. - JCL

\section{INFECTION \\ Viral exhaustion and epigenetics}

Expression of the inhibitory receptor PD-1 is tuned by epigenetic regulation of its gene during $\mathrm{CD} 8^{+} \mathrm{T}$ cell differentiation and chronic viral infection.

PD-1 is a cell-surface inhibitory receptor that is upregulated with $\mathrm{T}$ cell activation and 